Published in final edited form as:

Phys Med Biol. ; 63(21): 215027. doi:10.1088/1361-6560/aae7f1.

\title{
Discriminating lung adenocarcinoma from lung squamous cell carcinoma using respiration-induced tumor shape changes
}

\author{
Yi Lao ${ }^{1}$, John David ${ }^{1}$, Amin Mirhadi ${ }^{1}$, Natasha Lepore ${ }^{2}$, Howard Sandler ${ }^{1}$, Yalin Wang ${ }^{3}$, \\ Richard Tuli ${ }^{1}$, and Wensha Yang ${ }^{1,4,5}$ \\ ${ }^{1}$ Department of Radiation Oncology, Cedars Sinai Medical Center, Los Angeles, CA, United \\ States of America \\ 2Department of Radiology, Children's Hospital Los Angeles, Los Angeles, CA, United States of \\ America \\ ${ }^{3}$ School of Computing, Informatics, Decision Systems and Engineering, Arizona State University, \\ Tempe, AZ, United States of America \\ ${ }^{4}$ Department of Radiation Oncology, 8700 Beverly Blvd., Los Angeles, CA 90048, United States \\ of America
}

${ }^{5}$ Author to whom any correspondence should be Addressed.

\section{Abstract}

Based on 4D-CT, we aimed to characterize the pattern of morphological changes in lung tumors during respiration, and investigated its potential in non-invasively differentiating lung adenocarcinoma (AC) and squamous cell carcinoma (SCC).

\begin{abstract}
We applied a 3D surface analysis on 22 tumors (13 AC, 9 SCC) to investigate the tumor regional morphological fluctuations in response to respiration phases. Tumor surface vertices among ten respiratory phases were matched using surface-based registration, and the shape descriptors ( $\rho$ and $\operatorname{det} J$ ) were calculated and tracked across respiration stages in a regionally aligned scenario. Pairwise group comparisons were performed between lung AC and SCC subtypes, in terms of ratios of maximal shape changes as well as correlation coefficients between tumor shape and respiratory stage indicators from the lung.

AC type tumors had averaged larger surface measurements at exhale than at inhale, and these surface measurements were negatively correlated with lung volumes across respiratory stages. In contrast, SCC type tumors had averaged smaller surface measurements at exhale than at inhale, and the correlations with lung volumes were positive. The group differences in maximal shape changes as well as correlations were both statistically significant $(p<0.05)$.

We developed a non-invasive lung tumor sub-type detection pipeline based on respiration-induced tumor surface deformation. Significant differences in deformation patterns were detected between lung AC and SCC. The derived surface measurements may potentially serve as a new noninvasive imaging biomarker of lung cancer subtypes.
\end{abstract}




\section{Keywords}

4D-CT; non small cell lung cancer (NSCLC); adenocarcinoma; squamous cell carcinoma; tumor deformation; respiration; surfactant

\section{Introduction}

Lung cancer is one of the leading causes of cancerous deaths worldwide, with a five-year survival rate of 15\% (Siegel et al 2015, Fitzmaurice et al 2017). Adenocarcinoma (AC) and Squamous cell carcinoma (SCC) are two major types of non small cell lung cancer (NSCLC), with considerable prognostic differences in staging criteria, overall survival (Ichinose et al 1995, Suzuki et al 1999, Kawase et al 2011), and treatment options, particularly in the choices of chemotherapy regimens (Scagliotti et al 2011, Huang et al 2016). Therefore, pretreatment histopathological diagnosis of lung cancers is highly demanded in histologically adapted treatment to maximize therapeutic outcomes. To obtain a definitive pretreatment histopathological diagnosis, flexible bronchoscopy and CT-guided lung biopsy are two mainstream methods (de Margerie-Mellon et al 2016). The former is less sensitive to peripheral lung tumors, while the latter is contraindicated by significant coagulopathy, severe respiratory insufficiency and inadequate patient cooperation (Manhire et al 2003, Rivera et al 2013). With the development of imaging techniques, in vivo thoracic screening in high-risk populations has become possible, which facilitates early detections of lung abnormalities and leads to improved resectability and survival (Midthun 2013, Purandare and Rangarajan 2015). However, current imaging techniques have mainly focused on differentiating malignant lesions from benign nodules, providing inadequate characterization for tumor histological subtypes.

Although their exact originations are not clearly understood, lung AC and SCC are thought to arise from different cell types and thus have different tissue constitutions (Sutherland and Berns 2010, Hanna and Onaitis 2013). Tumors with AC histology often resemble the glandular structure and contain a significant amount of mucus, while tumors with SCC histology commonly constitute of flat cells that line inside major airways and are thus frequently seen with cavities (Hanna and Onaitis 2013, Purandare and Rangarajan 2015). Lung tumors, like other surrounding lung tissues, would also present varying levels of morphological changes with respiration (figure 1). Under the influence of internal airflow exchanges and surrounding tissue pressure fluctuations, these two types of lung tumors with distinct microenvironment and cellular constitutions may present different patterns of morphological changes during breathing. Therefore, quantifying the tumor shape changes across respiration phases has the potential to provide insights into the tumor tissue constitution and thus have further histologic implications.

Four-dimensional computed tomography (4D-CT) has been widely used in radiation therapy to monitor tumor respiratory motion to better define personalized tumor margins (Boldea et al 2008). Researchers have generally viewed tumors as rigid structures, and mainly considered the displacement of tumor center of mass (COM) during respiration. Only a few studies have attempted to investigate tumor shape deformation. In particular, to investigate 
whether lung tumors deform during respiration, $\mathrm{Wu}$ and colleagues compared the adequacy of lung tumor delineation using only rigid body transformation versus rigid plus deformable registration. In their work, the effect of respiration on lung tumor volumes was found to be primarily described by rigid translation, with a subtle, but observable improvement in accuracy by succeeding deformable registration (Wu et al 2009). Because the deformation is small, these morphological changes are often ignored by subsequent imaging analysis and are rarely associated with clinical diagnosis, which is the focus of the current study.

In this study, we track and quantify the regional tumor shape deformation based on 4D-CT. Our approach is twofold: firstly, to develop a 3D tumor shape based pipeline capable of detecting respiration-induced subtle morphological fluctuations; secondly, to evaluate the feasibility of using respiration-induced tumor deformation pattern as a new imaging biomarker for diagnosing two histological subtypes of lung cancers.

\section{Methods and materials}

\subsection{Subjects and data}

Under Institutional Review Board approval, 180 patients with histologically confirmed primary lung tumors or lung metastases were retrospectively solicited from our institutional database between the end of 2014 to the beginning of 2018. However, only 30 4D-CT images were retrieved for this study. 4D-CT were not mandatory in our institution during this period. It was either not ordered or discarded due to artifacts near the tumor for the rest 150 patients. After excluding the patients who did not go through biopsies or had non-adeno and nonsquamous lung metastases, 19 patients were finally enrolled in this study. All enrolled patients underwent 4DCT scans before radiation therapy. A total of 22 tumors (19 primaries and three metastatic) were identified in the enrolled patients. Tumors were biopsy confirmed and histologically discriminated into 11 primary AC, eight primary SCC, two metastatic AC, and one metastatic SCC. Patient demographic information and tumor characteristics are summarized in table 1 .

All 4D-CT scans were taken in a 16-slice GE Optima CT580 ${ }^{\mathrm{TM}}$ scanner with energy of 120 $\mathrm{kvp}$, current of $118 \mathrm{~mA}$, and slice thickness of $2.5 \mathrm{~mm}$. Each imaging session took an average of 15 min with a cumulative radiation dose of 5-10 cGy. A maximum of 3000 slices was collected in each scan to cover the whole lung or as much as allowed. 4D-CT scans were conducted in cine mode guided by Real-time Position Management $\left(\mathrm{RPM}^{\mathrm{TM}}\right)$ system (Varian Medical Systems, Palo Alto, CA) and reconstructed in AdvantageSim ${ }^{\mathrm{TM}}$ 4D software (GE Healthcare, Milwaukee, WI). To control the imaging quality, patient's breathing and movement were monitored in the clinical 4D-CT scan. If a patient achieved natural breathing and minimized non-breathing related body movement, we considered the artifacts were minimized. Additionally, board-certified clinical physicists evaluate every 4D-CT to determine stitching artifacts. The determinization of phase error was achieved through AdvantageSimTM software (GE), using RPMTM (Varian) as breathing surrogate. Scans with visible stitching artifacts or phase error greater than $5 \%$ were discarded and not included in our study. 


\subsection{Data preprocessing}

The data preprocessing pipeline is illustrated in figure 2. For each patient, the initial boundary of the gross tumor volume (GTV-init) and the affected lung (Lung-init) were manually segmented by a single radiation oncologist on the first 4D-CT phase (CT 0\%) corresponding to the end-of-inhalation (EOI). The intra-rater reliability score $\left(\frac{|A \cap B|}{|A \cup B|}\right)$ was 0.89 , in four participants (two for each lung cancer subtypes) at two different times spanning six weeks, in which A, B are segmentations. Tumor and Lung contours from the rest of the nine respiration phases were propagated through between-phase registrations. Specifically, CT images of phase 0 were first rigidly aligned to each of the following phases, and followed by nonlinear registration in the FMRIB software library (FSL) (Jenkinson et al 2012), to fully compensate internal movements and deformation. Linear and nonlinear registration matrices were combined to warp GTV-init and Lung-init and form GTV and Lung segmentations of the corresponding respiration phases. The contours were examined by the same radiation oncologist and edited when necessary.

Based on the resultant segmentation, 3D GTV meshes of each tumor for all ten phases were constructed using the marching cube algorithm (Lorensen and Cline 1987). To obtain regional correspondences between different respiratory phases, tumor surfaces from all phases were further matched using a surface-based constrained harmonic registration (Wang et al 2010, 2011). This step ensures that the surface tracking is limited to the tumor, excluding the influence of surrounding tissues.

\subsection{Statistical analysis}

We hypothesized that the tumor surface descriptors are sensitive to the morphological differences presented by lung SCC and AC. Three GTV shape descriptors were defined: structural-wise tumor volume $(V t)$, vertex-wise radial distance $(e)$ and determinant of Jacobian vectors $(\operatorname{det} J)$. $V t$ is an intuitive and coarse estimation of the shape changes, while the other two vertex-wise measurements probe into the complex tumor geometry. In particular, $\varrho$ is the distance between a surface vertex to the medial line of the structure, which directly estimates the thickness of a vertex, and captures the regional shape feature in the normal direction. A brief illustration of $\rho$ is shown in figure 3. $J$ is the Jacobian matrix of the deformation resulting from constrained harmonic registration. Suppose two triangle surface meshes with planar coordinates denoted by $v_{i}, w_{i}, i=1,2,3$. The discrete derivative map $J$ mapping $\left[v_{1}, v_{2}, v_{3}\right]$ to $\left[w_{1}, w_{2}, w_{3}\right]$ can be calculated by (Wang et al 2008, 2011, Shi et al 2013)

$$
J=\left[w_{3}-w_{1}, w_{2}-w_{1}\right]\left[v_{3}-v_{1}, v_{2}-v_{1}\right]^{-1} .
$$

The determinant of the Jacobian matrix $(\operatorname{det} J)$ for a surface vertex captures regional shape feature in surface area by describing the extent of necessary deformation to warp a specific local region to match its target.

Statistical analyses were conducted by two measurements: M1 measures maximal changes of the surface shape parameters between the EOI and EOE (end-of-exhalation) phases; M2 
measures the correlation coefficient between surface shape parameter fluctuations and breathing phases. Key steps of M1 and M2 were illustrated in figure 4. In M1, the intratumor averaged vertex-wise ratio of shape parameters $(\rho$, det $J$ ) were compared between EOI and EOE phases defined from the volume of the affected lung. Here, the respiration phases were determined using the volume of the tumor-bearing lung (excluding tumor tissues). In $\mathrm{M} 2$, the vertex-wise correlation between tumor shape descriptors $(\varrho$, det $J)$ and all respiration phases was performed. Specifically, at each tumor surface vertice, Pearson's correlation was calculated as follows:

$$
\begin{gathered}
R_{\rho-V_{\text {lung }}}=\frac{\operatorname{COV}\left(\rho, V_{\text {lung }}\right)}{\sigma_{\rho}{ }^{*} \sigma_{V_{\text {lung }}}} \\
R_{\operatorname{det} J-V_{\text {lung }}}=\frac{\operatorname{COV}\left(\operatorname{det} J, V_{\text {lung }}\right)}{\sigma_{\operatorname{det} J} * \sigma_{V_{\text {lung }}}}
\end{gathered}
$$

where $\varrho$, $\operatorname{det} J$ are two surface measurements, $V_{\text {lung }}$ is the tumor-bearing lung volume excluding tumor, and $C O V$ and $\sigma$ are covariance and standard deviation respectively. Significantly correlated vertices were extracted. The corresponding correlation coefficients were then averaged to determine to what extent and in which direction the tumor surface changes are correlated to lung movement.

On all measurements from M1 and M2, group comparisons were conducted between lung primary $\mathrm{AC}\left(\mathrm{AC}_{l}\right)$ and lung primary $\mathrm{SCC}\left(\mathrm{SCC}_{l}\right)$ groups, as well as all $\mathrm{AC}\left(\mathrm{AC}_{a}\right)$ and all $\mathrm{SCC}\left(\mathrm{SCC}_{a}\right)$ groups (tumors grouped regardless of primary or metastasis), using Student $t$ tests on respective parameters. Because the AC and SCC tumor locations are heterogeneous in terms of their proximity to central bronchi and their tumor-bearing lungs, the influence of tumor locations is analyzed by comparing surface descriptors of tumors in peripheral versus central and left versus right lung. In addition, the predictive power of the above-mentioned metrics was further evaluated using logistic regression followed by receiver operating characteristic (ROC) analysis.

\section{Results}

Figure 5 displays mean and standard deviations of measurements for all lung tumor subtypes using four different metrics: surface averaged $\varrho$ ratios $\left(\rho_{\text {ratio }}\right), \operatorname{det} J$ ratios $\left(\operatorname{det} J_{\text {ratio }}\right)$, averaged correlation coefficient over areas showing a significant association between shape and respiration phases $\left(R_{Q}-V_{\text {lung }}\right.$ and $\left.R_{\operatorname{det} J-V_{\text {lung }}}\right)$. Group comparison results based on these measurements are further summarized in table 2 .

As shown in figure 5, mean surface measurement ratios $\left(\rho_{\text {ratio }}\right.$ and $\left.\operatorname{det} J_{\text {ratio }}\right)$ for tumors with histology sub-type $\mathrm{AC}$ were greater than 1, showing expansion of tumor surfaces in the $\mathrm{EOE}$ 
compared to the EOI phase, while the same measurements for tumors with histology subtype SCC were less than 1, showing a contraction of tumor surfaces from EOI to EOE phase. The differences of $\varrho_{\text {ratio }}$ and $\operatorname{det} J_{\text {ratio }}$ between $\mathrm{AC}_{l}$ and $\mathrm{SCC}_{l}$ groups are both statistically significant $(p<0.05)$. The same significances were found in group comparisons between $\mathrm{AC}_{a}$ and $\mathrm{SCC}_{a}$. As a contrast, traditional measurements based on $V t$ detected no statistical significance in any of the group comparisons.

The differences in deformation trends were more evident by correlating surface descriptors with the whole breathing cycle in M2. After excluding surface areas either unchanged or uncorrelated to respiration, the remaining tumor surfaces with $\mathrm{AC}$ type $\left(\mathrm{AC}_{I}\right.$ and $\left.\mathrm{AC}_{a}\right)$ showed mean negative correlation to lung volumes, indicating tumor morphological changes opposite to that of the tumor-bearing lung. On the contrary, tumors with SCC type ( $\mathrm{SCC}_{l}$ and $\mathrm{SCC}_{a}$ ) showed mean positive correlation to the lung volume changes, indicating tumor morphological changes consistent to that of the tumor-bearing lung. In the group-wise comparisons shown in table 2, the differences of $R$ between $\mathrm{AC}_{l}$ and $\mathrm{SCC}_{l}$, as well as between $\mathrm{AC}_{a}$ and $\mathrm{SCC}_{a}$ are both statistically significant $(p<0.01)$. The differences in the four surface metrics for the tumors located in peripheral versus central or left versus right lung were not statistically significant.

The ROC curves of the five metrics in AC/SCC discrimination are presented in figure 6. As demonstrated by the respective AUCs, metrics derived from $\mathrm{M} 2\left(R_{\rho-V_{\text {lung }}, R} \operatorname{det} J-V_{\text {lung }}\right)$ performed the best in identifying AC tumors, either within the primary lung tumors (figure 6(a)), or in a mix of primary and metastatic lung tumors (figure 6(b)), both showed AUCs above 0.9. Consistent with results from group comparisons, compared to M2 derived metrics, M1 derived metrics $\left(\rho_{\text {ratio }}\right.$, det $J$ ratio $)$ were less efficient in AC identification, yielding AUCs of 0.81-0.89. As expected, for the same task, the performance of $V t_{\text {ratio }}$ was limited, with AUCs around 0.5 for both cases.

\section{Discussion}

\subsection{Methodological considerations}

4D imaging data reveal organ physiological and mechanical properties (Hegi-Johnson et al 2017). The advances of quantitative medical image analysis and the developments of registration algorithms have afforded the detection of tissue deformation during breathing. In recent years, the displacement field obtained from between-phase deformable registration has been utilized in 4D radiation therapy planning (Lim-Reinders et al 2017, Zhong et al 2017). By providing a more refined tumor delineation and an improved targeting accuracy in radiotherapy, deformable registration has shown to outperform registration based on translation and rotation only (Poulsen et al 2012, Ge et al 2014). However, the extent of tumor morphological changes with respiration is found to be small. Thus it is challenging to characterize using global radiomic features, such as short or long axes, sphericity, compactness and volume.

To obtain a quantitative assessment for regional morphological changes of normal organs or tumors, voxel-wise registration is a typical choice and has been effective in revealing shape 
deformation for organs with relatively large deformation, such as heart and lung (Weiss et al 2006, Li et al 2013). While for lung tumors, due to the relatively large translational displacement, the small shape changes can be overwhelmed by the variation of surrounding tissues within the region of interest. To eliminate the effect of surrounding tissues, one study extracted the 3D tumor from the original imaging space and measured the maximal tumor dimensional changes in three cardinal planes (Kyriakou and McKenzie 2012). However, this oversimplifies the actual tumor deformation reacting to heterogeneous mechanical environment and properties.

In this study, we modeled lung tumors using 3D surface meshes and tracked the morphological fluctuations in a regionally aligned condition. We used two metrics to capture local shape changes: $\rho$ and $\operatorname{det} J$. These two metrics characterize vertex-wise geometric features in two main aspects - the normal direction and the surface area, and have been shown to efficiently reveal small morphological alterations (Lao et al 2014, 2017). Surfacebased constrained harmonic registration affords vertex-to-vertex correspondences, which is a key prerequisite to the regional analysis and has been validated in analyses of brain anatomic abnormalities (Wang et al 2011, Lao et al 2014, 2017). Using 3D surface models, respiration-related tumor morphological changes are monitored on all surface vertices, allowing the analysis of shape expansions and contractions on the complete tumor surfaces, instead of the limited three cardinal planes. This allows a more accurate characterization of the intrinsically heterogenous lung tumor that exhibits non-uniform surface changes with respiration.

In addition to directly measuring the extent of tumor changes in M1, the correlation based method (M2) denotes how 'responsive' the tumor changes with respiration and is less affected by the absolute tumor deformation. Combining the results from M1 and M2, possible biases of tumor locations in histologic-wise group comparisons are also mitigated. Moreover, using correlations between tumor surface parameters and lung measurements, we are able to extract the changes that are truly associated with respiration. This further reduces the influence of irrelevant tumor surface alterations from non-respiratory activities, such as pulsations of heart and vessels, and inevitable image artifacts from 4D-CT images.

\subsection{Pathological considerations}

Although the exact cellular mechanism underpinning the observed differences is beyond the scope of our study, the different deformation patterns presented by the two histologic subtypes indicate contrasting tumor tissue constitutions, which may be further traced to the altered regulation of pulmonary surfactant. Formed by type-II alveoli cells, pulmonary surfactant is a surface active protein capable of reducing alveolar surface tension and preventing atelectasis (Zuo et al 2008). Growing evidence has linked lung AC to the mutations of type II surfactant secreting alveoli cells (Xu et al 2012), while the gene expression patterns of lung SCC are thought to originate from tracheal basal cell progenitors (Sutherland and Berns 2010). Close examination of peripheral-blood samples of lung cancer patients reported significantly higher expression rate of Pro-Surfactant B in lung AC type than that in SCC type as well as healthy controls (He et al2017), while no difference was detected between SCC type and controls (Sin et al 2013). As a result, abnormal regulation of 
surfactant in lung AC tumors would lead to a breakage of regular lung homeostasis and may induce a 'delay' in tumor tissues that disaccord the regular rhythm of rising and falling in normal lung tissues. However, due to the retrospective nature of this study, individual surfactant levels were not available. Therefore, this preliminary hypothesis needs further validation. For instance, a correlation between the 'delay' in AC tumors and patient's pulmonary surfactant levels, would help elucidate how the alterations in surfactant levels may result in changes in tumor deformations.

As cancer-initiating cells are likely to inherit the characteristic attributes of normal tissue stem cells, meta-static AC and SCC tumor cells may exhibit similar tissue constitutions as primary tumors and thus have similar deformation patterns in respiration. Our group analyses on $\mathrm{AC}_{a}$ and $\mathrm{SCC}_{a}$ groups are consistent with these cellular properties. Nonetheless, this should be validated in a larger cohort study, since only three metastatic tumors were included in this study. In addition, as shown in figure 5, the averaged correlation coefficients over significant areas in the AC group only trend in the negative direction, implying mixed positive and negative correlations presented in AC tumor surfaces, with the negative ones being dominant. This can be attributed to the tumor heterogeneity. Although the tumor surface presents an overall trend of asynchronization to lung expansions and contractions, different areas of the same tumor may still present different behaviors, possibly due to the uneven distribution of surfactant within tumors, or blockage of airflow tubular with surrounding airways. However, these explanations should be taken with caution, given the small sample size the absence of direct tissue surfactant data. Future large-scale studies with more biological examination will be needed to validate the hypothesis generated here.

\subsection{Limitations and future directions}

There are several limitations in our study. First, the small sample size, especially for the SCC group, has limited the statistical power in our results. For the same reason, the compounding effects of patients' age, gender, and tumor staging on respiration-induced tumor deformation cannot be excluded. Second, tumor contour delineation was performed on $4 \mathrm{D}-\mathrm{CT}$, which were subject to binning artifacts. Although our correlation based methods have potentially removed considerable deformation caused by random image artifacts, the effect of image artifacts cannot be completely eliminated. Future advanced reconstruction protocols shall be integrated into the scan to achieve optimized projection binning (Werner et al 2017). Third, respiration-induced shape changes were tracked in limited phases ( $n=$ 10), which may be inadequate to describe the possible delay between lung and tumor deformation. In the future, the surface morphology tracking pipeline can be further validated on larger datasets with higher temporal resolution.

\section{Conclusions}

Based on ten binned four D-CT, we developed a tumor surface morphology tracking pipeline to characterize the respiration-induced tumor deformation. The derived surface measurements successfully detected significant differences in deformation patterns between histologic subtypes of AC and SCC. To our knowledge, this is the first study to show the diagnostic value of the respiration-induced $4 \mathrm{D}$ deformation. The developed surface 
measurements can potentially serve as new non-invasive imaging biomarker discriminating lung AC and SCC subtypes, while these findings need to be validated in a larger study.

\section{References}

Boldea V, Sharp GC, Jiang SB and Sarrut D 2008 4d-ct lung motion estimation with deformable registration: quantification of motion nonlinearity and hysteresis Med. Phys 35 1008-18 [PubMed: 18404936]

de Margerie-Mellon C, de Bazelaire C and de Kerviler E 2016 Image-guided biopsy in primary lung cancer: why, when and how Diagn. Intervent. Imaging 97 965-72

Fitzmaurice C et al. 2017 Global, regional, and national cancer incidence, mortality, years of life lost, years lived with disability, and disability-adjusted life-years for 32 cancer groups, 1990 to 2015: a systematic analysis for the global burden of disease study JAMA Oncol3 524-48 [PubMed: 27918777]

Ge Y, O'Brien RT, Shieh C-C, Booth JT and Keall PJ 2014 Toward the development of intrafraction tumor deformation tracking using a dynamic multi-leaf collimator Med. Phys 41061703 [PubMed: 24877798]

Hanna JM and Onaitis MW 2013 Cell of origin of lung cancer J. Carcinog 12

He Y, Jiang Z, Tong F, Li M, Yin X, Hu S and Wang L 2017 Experimental study of peripheral-blood pro-surfactant protein B for screening non-small cell lung cancer Acta Cirurg. Bras 32 568-75

Hegi-Johnson F, Keall P, Barber J, Bui C and Kipritidis J 2017 Evaluating the accuracy of 4D-CT ventilation imaging: first comparison with technegas spect ventilation Med. Phys 44 4045-55 [PubMed: 28477378]

Huang T, Li J, Zhang C, Hong Q, Jiang D, Ye M and Duan S 2016 Distinguishing lung adenocarcinoma from lung squamous cell carcinoma by two hypomethylated and three hypermethylated genes: a meta-analysis PLoS One 11 e0149088 [PubMed: 26862903]

Ichinose Y, Yano T, Asoh H, Yokoyama H, Yoshino I and Katsuda Y 1995 Prognostic factors obtained by a pathologic examination in completely resected non-small-cell lung cancer: an analysis in each pathologic stage J. Thoracic Cardiovascular Surg 110 601-5

Jenkinson M, Beckmann CF, Behrens TE, Woolrich MW and Smith SM 2012 FSL NeuroImage 62 782-90 [PubMed: 21979382]

Kawase A, Yoshida J, Ishii G, Nakao M, Aokage K, Hishida T, Nishimura M and Nagai K 2011 Differences between squamous cell carcinoma and adenocarcinoma of the lung: are adenocarcinoma and squamous cell carcinoma prognostically equal? Japan. J. Clin. Oncol42 18995 [PubMed: 22210923]

Kyriakou E and McKenzie D 2012 Changes in lung tumor shape during respiration Phys. Med. Biol 57 919 [PubMed: 22290510]

Lao Y, Dion L-A, Gilbert G, Bouchard MF, Rocha G, Wang Y, Leporé N and Saint-Amour D 2017 Mapping the basal ganglia alterations in children chronically exposed to manganese Sci. Rep 7 41804 [PubMed: 28155922]

Lao Y, Wang Y, Shi J, Ceschin R, Nelson MD, Panigrahy A and Leporé N 2014 Thalamic alterations in preterm neonates and their relation to ventral striatum disturbances revealed by a combined shape and pose analysis Brain Struct. Funct 221 487-506 [PubMed: 25366970]

Li M, Castillo E, Zheng X-L, Luo H-Y, Castillo R, Wu Y and Guerrero T 2013 Modeling lung deformation: a combined deformable image registration method with spatially varying Young's modulus estimates Med. Phys 40081902 [PubMed: 23927316]

Lim-Reinders S, Keller BM, Al-Ward S, Sahgal A and Kim A 2017 Online adaptive radiation therapy Int. J. Radiat. Oncol. Biol. Phys 99 994-1003 [PubMed: 28916139]

Lorensen WE and Cline HE 1987 Marching cubes: a high resolution 3D surface construction algorithm ACM Siggraph Computer Graphics vol 21 (New York: ACM) pp 163-9

Manhire A, Charig M, Clelland C, Gleeson F, Miller R, Moss H, Pointon K, Richardson C and Sawicka E 2003 Guidelines for radiologically guided lung biopsy Thorax 58 920-36 [PubMed: 14586042] 
Midthun DE. 2013; Early diagnosis of lung cancer. F1000Prime Rep. 5:12. [PubMed: 23585930]

Poulsen PR, Fledelius W, Cho B and Keall P 2012 Image-based dynamic multileaf collimator tracking of moving targets during intensity-modulated arc therapy Int. J. Radiat. Oncol. Biol. Phys 83 e265-71 [PubMed: 22401924]

Purandare NC and Rangarajan V 2015 Imaging of lung cancer: implications on staging and management Indian J. Radiol. Imaging 25109 [PubMed: 25969634]

Rivera MP, Mehta AC and Wahidi MM 2013 Establishing the diagnosis of lung cancer CHEST J 143 e142S-65S

Scagliotti G, Brodowicz T, Shepherd FA, Zielinski C, Vansteenkiste J, Manegold C, Simms L, Fossella F, Sugarman K and Belani CP 2011 Treatment-by-histology interaction analyses in three phase III trials show superiority of pemetrexed in nonsquamous non-small cell lung cancer J. Thoracic Oncol 6 64-70

Shi J et al. 2013 Surface fluid registration of conformal representation: application to detect disease burden and genetic influence on hippocampus NeuroImage 78 111-34 [PubMed: 23587689]

Siegel RL, Miller KD and Jemal A 2015 Cancer statistics, 2015 CA Cancer J. Clin 65 5-29 [PubMed: 25559415]

Sin DD. et al. 2013; Pro-surfactant protein B as a biomarker for lung cancer prediction. J. Clin. Oncol. 31:4536. [PubMed: 24248694]

Sutherland KD and Berns A 2010 Cell of origin of lung cancer Mol. Oncol 4 397-403 [PubMed: 20594926]

Suzuki K, Nagai K, Yoshida J, Nishimura M, Takahashi K, Yokose T and Nishiwaki Y 1999 Conventional clinicopathologic prognostic factors in surgically resected nonsmall cell lung carcinoma Cancer 86 1976-84 [PubMed: 10570421]

Wang Y, Song Y, Rajagopalan P, An T, Liu K, Chou Y-Y, Gutman B, Toga AW and Thompson PM 2011 Surface-based TBM boosts power to detect disease effects on the brain: an $n=804$ ADNI study NeuroImage 56 1993-2010 [PubMed: 21440071]

Wang Y, Yin X, Zhang J, Gu X, Chan T, Thompson PM and Yau S-T 2008 Brain mapping with the Ricci flow conformal parameterization and multivariate statistics on deformation tensors 2nd MICCAI Workshop on Mathematical Foundations of Computational Anatomy pp 36-47

Wang Y, Zhang J, Gutman B, Chan TF, Becker JT, Aizenstein HJ, Lopez OL, Tamburo RJ, Toga AW and Thompson PM 2010 Multivariate tensor-based morphometry on surfaces: application to mapping ventricular abnormalities in HIV/AIDS NeuroImage 49 2141-57 [PubMed: 19900560]

Weiss JA, Veress AI, Gullberg GT, Phatak N, Sun Q, Parker D and Rabbitt RD 2006 Strain measurement using deformable image registration Mechanics of Biological Tissue (Berlin: Springer) pp 489-501

Werner R, Hofmann C, Mücke E and Gauer T 2017 Reduction of breathing irregularity-related motion artifacts in low-pitch spiral 4D-CT by optimized projection binning Radiat. Oncol 12100 [PubMed: 28629434]

Wu J, Lei P, Shekhar R, Li H, Suntharalingam M and D'Souza WD 2009 Do tumors in the lung deform during normal respiration? An image registration investigation Int. J. Radiat. Oncol. Biol. Phys 75 268-75 [PubMed: 19515506]

Xu X, Rock JR, Lu Y, Futtner C, Schwab B, Guinney J, Hogan BL and Onaitis MW 2012 Evidence for type II cells as cells of origin of K-Ras induced distal lung adenocarcinoma Proc. Natl Acad. Sci 109 4910-5 [PubMed: 22411819]

Zhong H, Siddiqui SM, Movsas B and Chetty IJ 2017 Evaluation of adaptive treatment planning for patients with non-small cell lung cancer Phys. Med. Biol 624346 [PubMed: 28072395]

Zuo YY, Veldhuizen RA, Neumann AW, Petersen NO and Possmayer F 2008 Current perspectives in pulmonary surfactant-inhibition, enhancement and evaluation Biochim. Biophys. Acta 1778 1947-77 [PubMed: 18433715] 


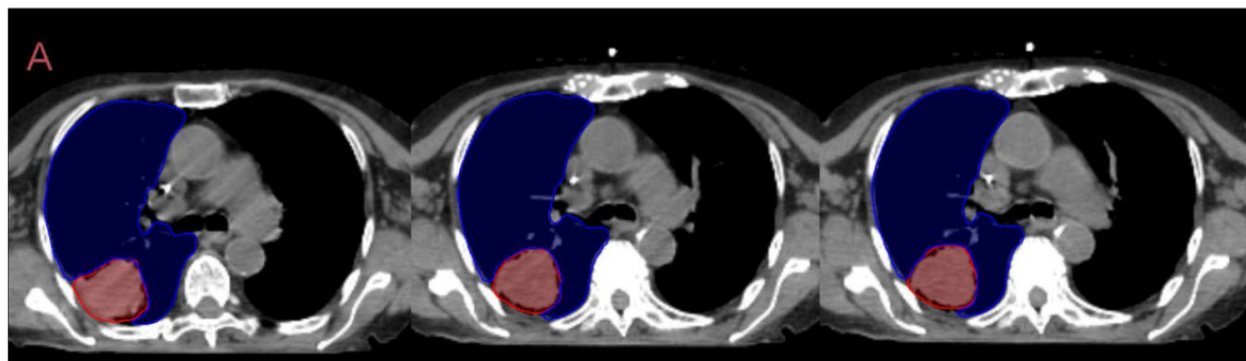

Phase $10 \%$

-

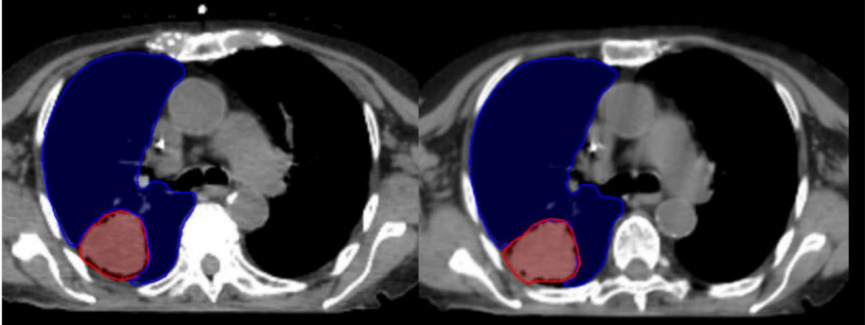

Phase $70 \%$

Phase 90\%
Phase 50\%

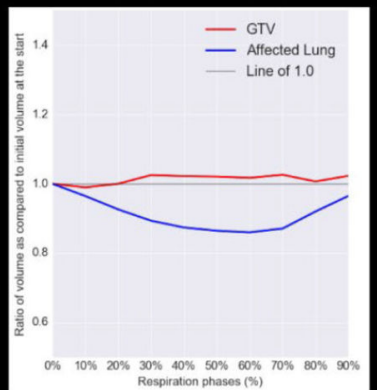

B

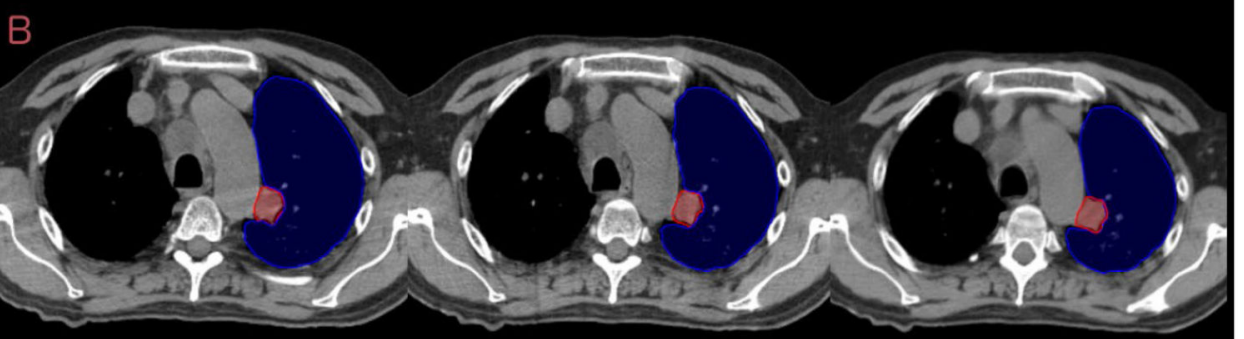

Phase 10\%

Phase 30\%

Phase 50\%

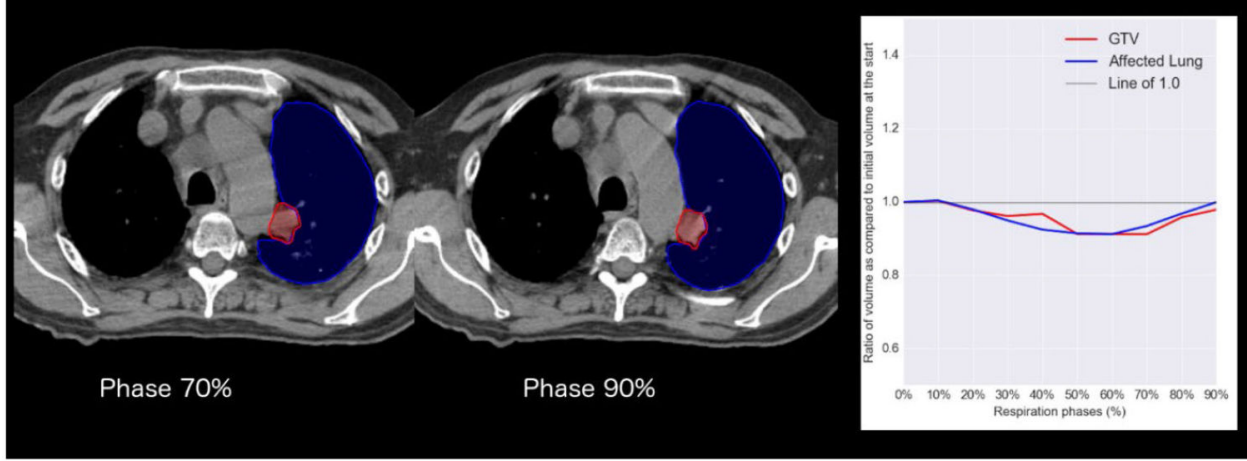

Figure 1.

Examples of axial 4D-CT for a patient with lung AC (A) and another with lung SCC (B), as

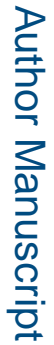
well as their corresponding tumor and lung volume trajectories across respiratory phases. Tumor and lung contours were highlighted in red and blue, respectively. 


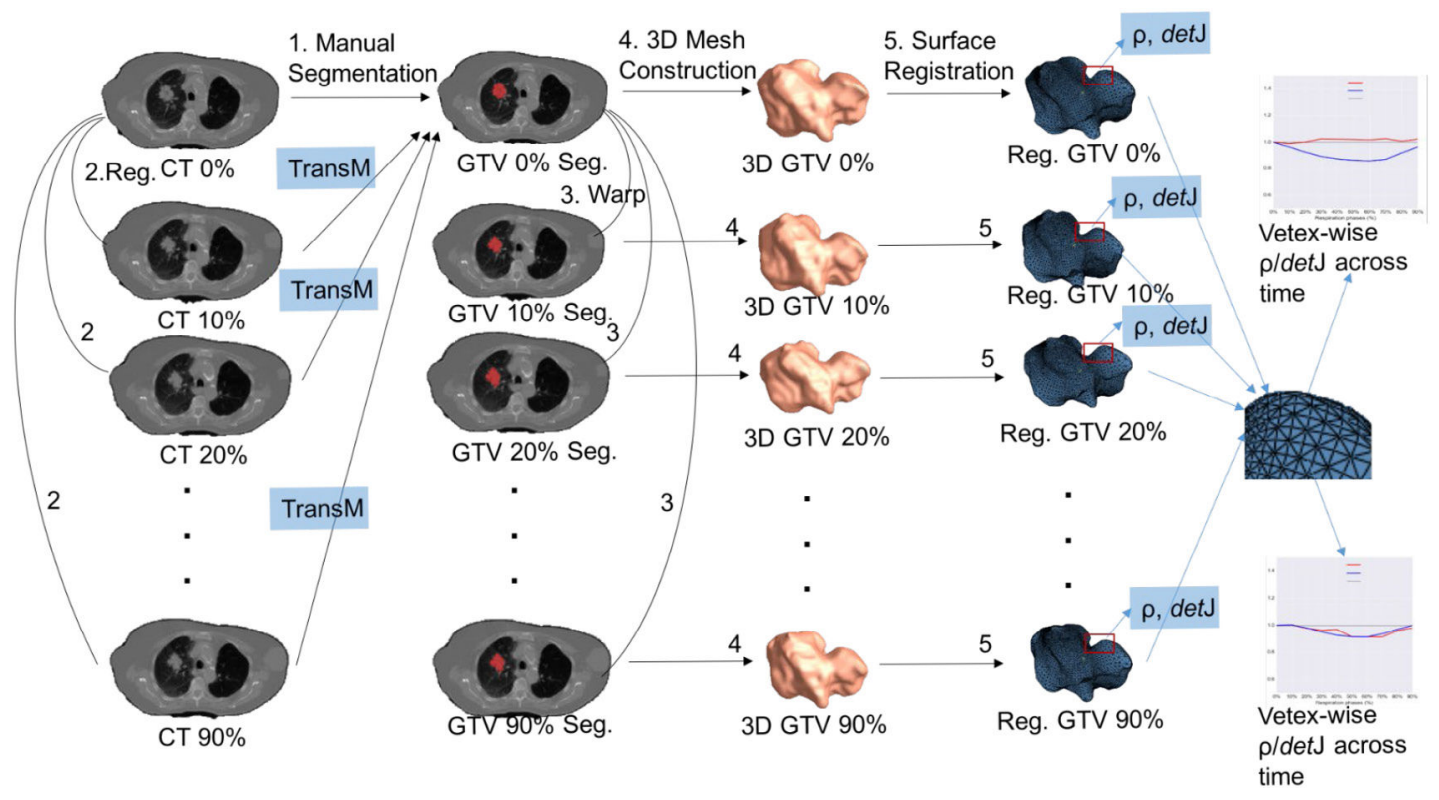

Figure 2.

Data preprocessing pipeline: from the registration based delineation of tumor contours to the construction of time-series of tumor shape parameters. For each of the subject, processing steps include: (1) manual segmentation of tumor boundaries on CT 0\% (EOI); (2) linear and nonlinear registration (reg.) between CT $0 \%$ and CT from other respiration phases; (3) applying registration resulted in a transformation matrix (TransM) on tumor segmentation from CT $0 \%$; (4) 3D surface modal construction based on the above-obtained tumor segmentations; (5) surface-based registration and generation of vertex-wise shape parameters. Tumor surface parameters from all the phases were assembled to construct an intra-subject time-series model of tumor shape for later statistical analysis. 


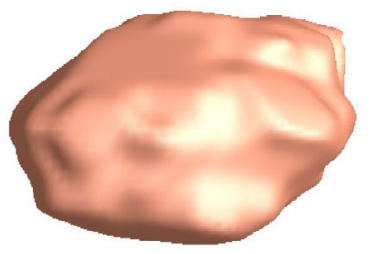

A

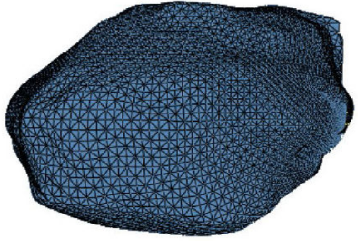

$\mathrm{B}$

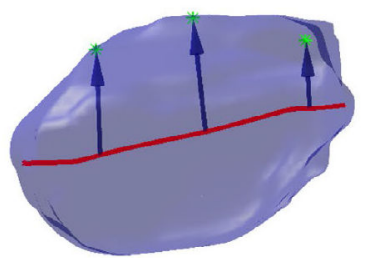

C

Figure 3.

Example of a 3D tumor model (A), its surface mesh (B), and a brief illustration of vertexwise radius $\varrho(\mathrm{C})$. In $\mathrm{C}$, the midline of the tumor model is shown in red. Three example surface vertices and their corresponding radius are marked in green stars and blue arrows, respectively. 
A

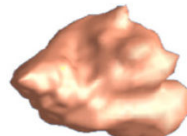

Tumor Surface Model (End-of-inhale)

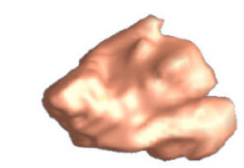

Tumor Surface Model (End-of-exhale)

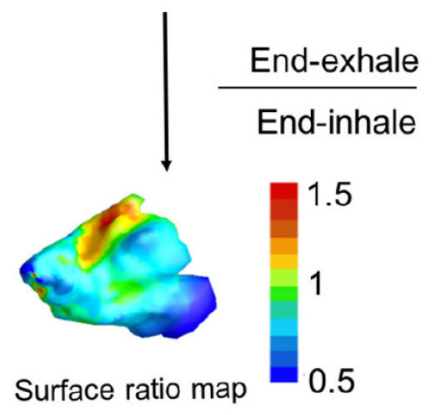

B
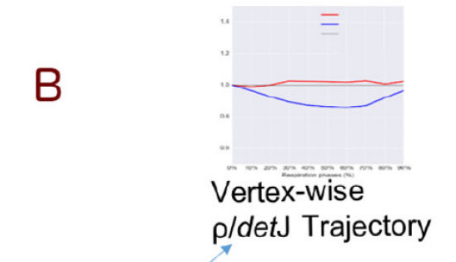

p/detJ Trajectory

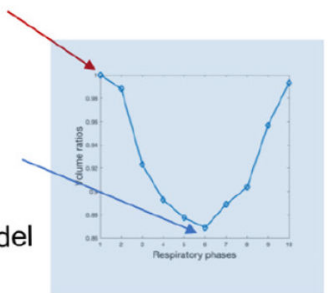

Tumor Surface Model

Correlation

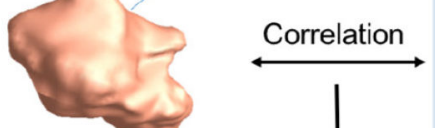

$\mathrm{P} \quad \mathrm{R}$
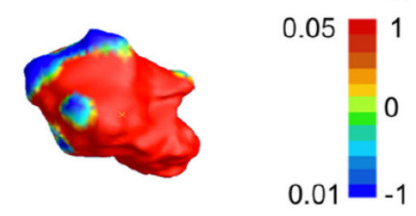

Surface $p$-value map

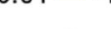

Surface correlation coefficient map

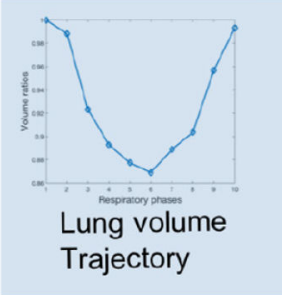

Correlation coefficient with significance

Figure 4.

Illustrations of statistical analysis methods M1 (A) and M2 (B). In M1, for each subject, a stage with least lung volume in the ten phases is identified as the end-exhale stage. Surface parameters between the end-exhale and the end-inhale stage are compared in the vertexwise, and an intra-tumor averaged ratio are calculated for group comparisons. In M2, correlation analysis is conducted between time-series of tumor shape parameters $(\varrho$, det $J)$ and respiration stage representers $\left(V_{\text {lung }}\right.$ ). Surface correlation coefficients with significance correlations $(p<0.05)$ are then extracted and averaged for group comparisons. 


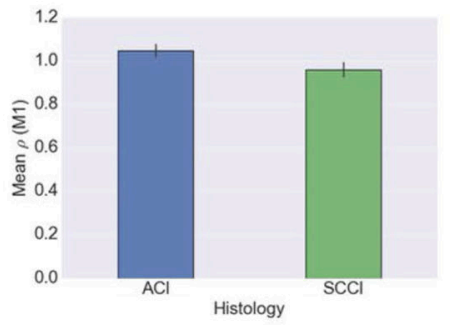

(a) $\rho_{\text {ratio }}-\mathrm{Lv}$ (primary)

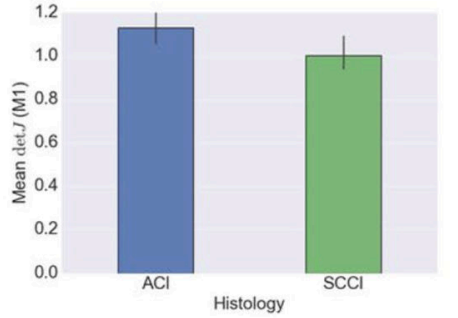

(c) $\operatorname{det} J_{\text {ratio }}-\mathrm{Lv}$ (primary)

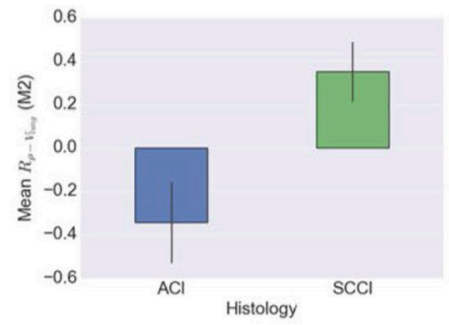

(e) $R_{\rho-V_{\text {lung }}}$ (primary)

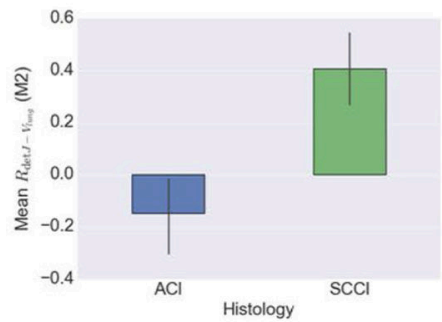

(g) $R_{\text {det } J-V_{\text {lung }}}$ (primary)

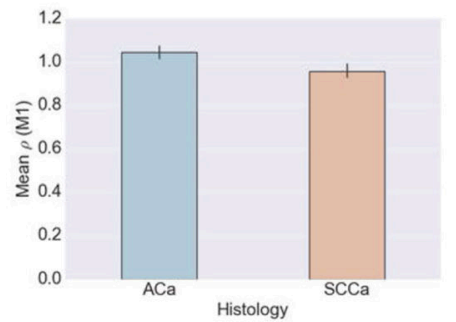

(b) $\rho_{\text {ratio }}-\mathrm{Lv}$ (all)

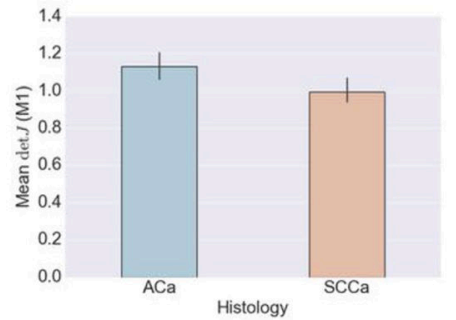

(d) $\operatorname{det} J_{\text {ratio }}-\mathrm{Lv}$ (all)

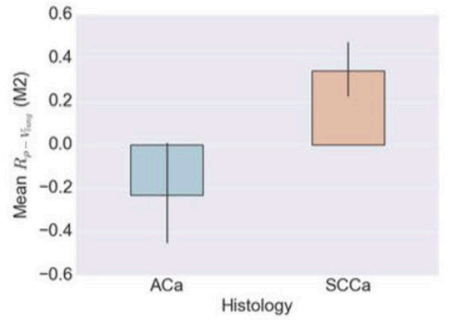

(f) $R_{\rho-V_{\text {lung }}}$ (all)

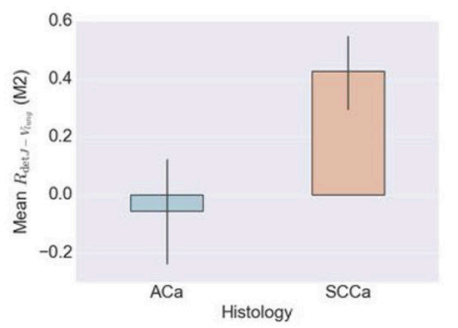

(h) $R_{\operatorname{det} J-V_{\text {lung }}}$ (all)

Figure 5.

Bar plots of respective measurements from primary (left column) and all (right column) AC and SCC tumors in four different methods: surface averaged $\varrho$ ratios (a) and (b) and $\operatorname{det} J$ ratios (c) and (d); averaged correlation coefficient between shape parameters $(\rho$, det $J)$ and respiration phases ( $V_{\text {lung }}$ ) (e)-(h). 


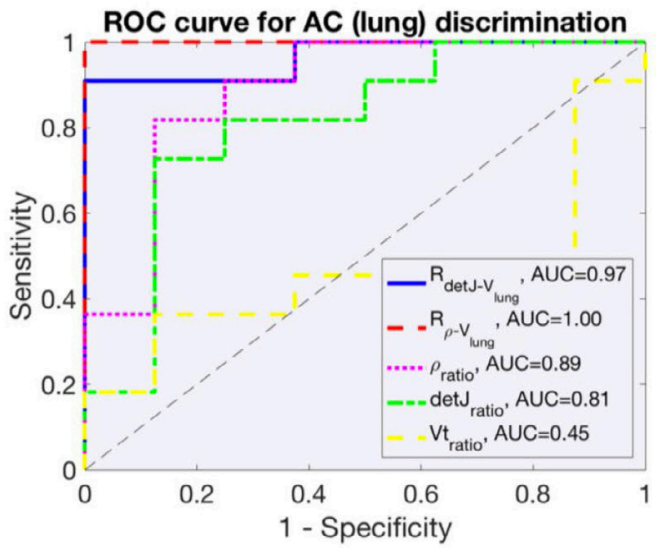

(a) Prediction on primary lung tumors.

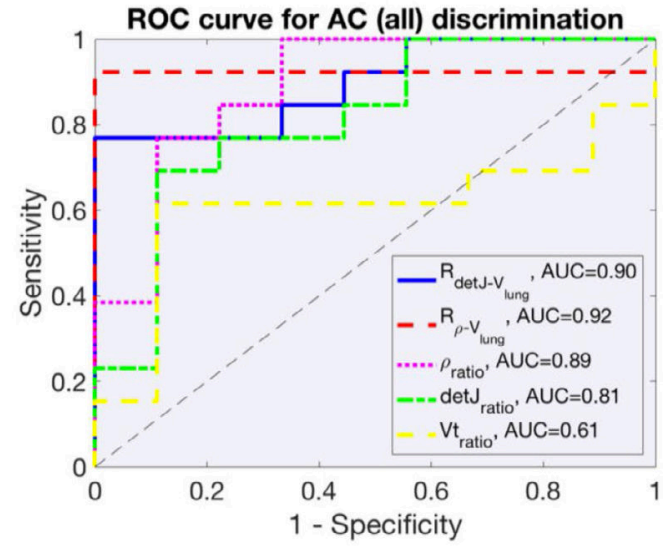

(b) Prediction on all lung tumors.

Figure 6.

ROC curves for the identification of AD in primary lung tumors (a) and all lung tumors (b), using the five different metrics shown in table 2. 
Table 1.

Patient and tumor characteristics.

\begin{tabular}{|c|c|c|c|c|c|c|}
\hline \multicolumn{7}{|c|}{ Panel A: patient characteristics } \\
\hline Patient ID & Age(y) & Gender & Tumor histology $^{a}$ & Location $^{b}$ & Affected lung & Tumor volume ${ }^{c}(\mathrm{cc})$ \\
\hline 01 & 71 & $\mathrm{~F}$ & Lung AC & $\mathrm{C} / \mathrm{B}$ & Right & 18.43 \\
\hline 02 & 87 & M & Lung SCC & $\mathrm{C} / \mathrm{U}$ & Left & 3.38 \\
\hline 03 & 90 & $\mathrm{~F}$ & Lung AC & $\mathrm{P} / \mathrm{U}$ & Right & 19.10 \\
\hline 04 & 80 & $\mathrm{~F}$ & Lung SCC & $\mathrm{C} / \mathrm{U}$ & Left & 21.37 \\
\hline 05 & 69 & M & Lung AC & $\mathrm{P} / \mathrm{U}$ & Left & 1.65 \\
\hline 06 & 89 & M & Lung AC & $\mathrm{P} / \mathrm{U}$ & Right & 42.54 \\
\hline 07 & 72 & M & Lung AC & $\mathrm{P} / \mathrm{U}$ & Left & 31.84 \\
\hline 08 & 64 & M & Lung AC & $\mathrm{P} / \mathrm{U}$ & Left & 1.02 \\
\hline 09 & 77 & $\mathrm{~F}$ & Lung AC & $\mathrm{P} / \mathrm{U}$ & Left & 9.54 \\
\hline 10 & 68 & M & Lung SCC & $\mathrm{C} / \mathrm{U}$ & Left & 20.93 \\
\hline 11 & 65 & $\mathrm{~F}$ & Mets AC & $\mathrm{C} / \mathrm{U}$ & Right & 11.58 \\
\hline 12 & 66 & M & Lung AC & $\mathrm{C} / \mathrm{U}$ & Left & 163.64 \\
\hline 13 & 81 & $\mathrm{~F}$ & Lung AC & $\mathrm{C} / \mathrm{U}$ & Left & 14.56 \\
\hline 14 & 58 & M & Lung AC & $\mathrm{C} / \mathrm{U}$ & Right & 1.42 \\
\hline 15 & 84 & M & Mets SCC & $\mathrm{P} / \mathrm{U}$ & Left & 3.46 \\
\hline 16 & 70 & M & Lung SCC & $\mathrm{C} / \mathrm{U}$ & Left & 72.30 \\
\hline \multirow[t]{2}{*}{$17^{d}$} & 72 & $\mathrm{~F}$ & Lung SCC & $\mathrm{C} / \mathrm{U}$ & Right & 33.61 \\
\hline & & & Lung SCC & $\mathrm{C} / \mathrm{U}$ & Right & 54.02 \\
\hline \multirow[t]{2}{*}{$18^{d}$} & 84 & M & Lung AC & $\mathrm{P} / \mathrm{U}$ & Right & 3.24 \\
\hline & & & Lung AC & $\mathrm{P} / \mathrm{U}$ & Right & 2.10 \\
\hline \multirow[t]{2}{*}{$19^{d}$} & 72 & M & Lung SCC & $\mathrm{P} / \mathrm{B}$ & Right & 1.25 \\
\hline & & & Lung SCC & $\mathrm{P} / \mathrm{B}$ & Right & 7.16 \\
\hline \multicolumn{7}{|c|}{ Panel B: summary of tumor characteristics } \\
\hline Groups & Histology & Peripheral/central & Upper/bottom & Affected lung & Volume $^{e}(\mathrm{cc})$ & \\
\hline $\mathrm{AC}_{I}(n=11)$ & $11 \mathrm{AC}$ & $6 \mathrm{P} 5 \mathrm{C}$ & 11U1B & $5 \mathrm{~L} 6 \mathrm{R}$ & $25.20(47.57)$ & \\
\hline $\operatorname{SCC}_{I}(n=8)$ & $8 \mathrm{SCC}$ & $2 \mathrm{P} 6 \mathrm{C}$ & $6 \mathrm{U} 2 \mathrm{~B}$ & $4 \mathrm{~L} 4 \mathrm{R}$ & $26.75(25.38)$ & \\
\hline $\operatorname{Mets}(n=3)$ & 2AC1SCC & $2 \mathrm{P} 1 \mathrm{C}$ & $3 \mathrm{U}$ & $2 \mathrm{~L} 1 \mathrm{R}$ & $15.63(14.62)$ & \\
\hline
\end{tabular}

${ }^{a}$ Adeno (AC), squamous (SCC).

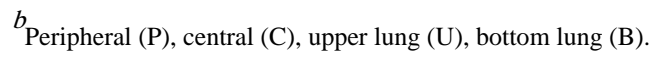

$c$ Volumes are measured at the end of the inhalation stage.

${ }_{\text {Patients with two primary tumors. }}$

e Mean (standard deviation). 
Table 2.

Summary of statistical group comparison results.

\begin{tabular}{|c|c|c|c|c|}
\hline & $\begin{array}{l}\mathrm{AC}_{l} \text { versus } \mathrm{SCC}_{l} \\
11 / 8\end{array}$ & $\begin{array}{l}\mathrm{AC}_{a} \text { versus } \mathrm{SCC}_{a} \\
13 / 9\end{array}$ & $\begin{array}{l}P \text { versus } C \\
11 / 11\end{array}$ & $\begin{array}{l}L \text { versus } R \\
11 / 11\end{array}$ \\
\hline$V_{t_{\text {ratio }}}$ & 0.8942 & 0.8672 & 0.1928 & 0.5474 \\
\hline$\rho_{\text {ratio }}$ & $0.0027^{b}$ & $0.0013^{b}$ & 0.6357 & 0.6720 \\
\hline $\operatorname{det}^{J}$ ratio & $0.0409^{a}$ & $0.0232^{a}$ & 0.4063 & 0.9074 \\
\hline$R_{\rho}-V_{\text {lung }}$ & $0.0001^{b}$ & $0.0013^{b}$ & 0.9793 & 0.9664 \\
\hline$R_{\operatorname{det} J-V_{\text {lung }}}$ & $0.0001^{b}$ & $0.0009^{b}$ & 0.7649 & 0.5505 \\
\hline
\end{tabular}

Significant comparisons are marked using

${ }^{a}$ for $p<0.05$, and

$b_{\text {for } p}<0.01$ 